\title{
A Novel Design Methodology for the Mixed-Domain Optimization of a MEMS Accelerometer
}

\author{
Murat PAK ${ }^{1}$, Francisco V. FERNANDEZ ${ }^{2}$, Gunhan DUNDAR ${ }^{1 .}$ \\ 1. Department of Electrical and Electronics Engineering, Bogazici University, Istanbul, Turkey. \\ E-Mails:murat.pak@boun.edu.tr,dundar@boun.edu.tr \\ 2. IMSE, CSIC and University of Sevilla, Spain. \\ E-Mail: pacov@imse-cnm.csic.es
}

\begin{abstract}
This paper proposes a novel optimization-based design methodology that can be used for mixed-domain synthesis of MEMS accelerometers. Several problems have been identified with existing methodologies and comparative experiments that demonstrate the superiority of the proposed methodology are performed. Highly accurate analytical models of the MEMS accelerometer device have been used for the evaluation of the MEMS sensor performances in the mixed-domain optimization. The circuit level simulations, on the other hand, are based on an electrical simulator, e.g., Hspice. The implemented methodology has been tested on the optimization of a MEMS accelerometer that includes a capacitive MEMS sensor and an analog read-out circuitry.
\end{abstract}

\section{I .INTRODUCTION}

Complex trade-off exploration in MEMS design, still requires a lot of expert knowledge, which implies long design times and increased cost especially due to the physical heterogeneity of the MEMS systems. Consequently, trial and error approaches are still widely used for MEMS design. Design automation, on the other hand, dramatically shortens the design time and also enables optimal designs, which even an expert designer cannot easily achieve. Among automated design methodologies, optimizationbased ones are especially promising for complex mixed-domain systems such as MEMS.

Design of MEMS comprises different domains like the mechanical MEMS sensor and the electronic read-out circuitry for signal conditioning, therefore, being a challenging problem. Industry has traditionally followed ad-hoc approaches based on separately designing the MEMS sensor and the circuitry by different engineering teams and, then, combining parts. This can either be achieved by using a pre-designed MEMS sensor and, then, implementing the electronic circuitry, or by composing an optimal sensor design with an optimal circuit design in order to obtain a so-called optimal system performance. However, combining these separately designed blocks is problematic for several reasons. A first problem is that inappropriate partitioning of the system specifications among the mechanical and electronic parts might lead to non-optimal system performance. Another problem, that can appear during the composition of independently designed blocks, is the risk of violating some system level constraints. For example, the sensor itself can be designed to satisfy a certain measurement range, and its composition with an inappropriate circuit design may lead to a measurement range of the system that does not satisfy the constraints due to an inappropriate level of amplification. Thus, the coupling of the separately designed mechanical and electronic devices is a challenge. Hence, a design methodology that simultaneously considers both the mechanical and the electronic parts in order to develop a multi-domain MEMS synthesizer is needed.

Several optimization-based approaches have been developed for the synthesis of MEMS sensors. Some of them are focused on the optimization of the MEMS device by either performing device level simulations of the MEMS sensors within an optimization loop $[1,2]$ or using accurate analytical models to speed up the synthesis process $[3,4,5]$.

Some other efforts $[6,7,8]$ have been devoted to multi-domain optimization of MEMS, mostly being inspired by VLSI hierarchical design methodologies, that aim at decomposing the mixed-domain design problem into smaller optimization subproblems, like the sensor itself and the input stage electronics. The approach in [6] focuses on developing a design methodology for the MEMS sensor where a mixed-domain circuit simulation is performed to obtain transient simulation results of the complete MEMS. However, the synthesis results focus on the MEMS sensor only instead of the whole system. In [7], the MEMS sensor and the circuit are designed at the behavioral level to enable their joint simulation. The design parameters at the circuit level are only a few high-level parameters such as the modulation voltage. None of the circuit level design parameters, like the transistor sizes or the values of the passive devices, have been used. Hence, only a high-level architectural description, dedicated to a sigma-delta control loop, is defined, instead of developing a device-level solution that is applicable to any MEMS. In [8], on the other hand, a top-down design methodology, which is based on optimizing the sensor first and, then, the circuitry using the optimal sensor design has been reported. This type of methodology has several drawbacks that cause non-optimal system designs as explained in Section 4. Therefore, to the best of our knowledge, no generic design methodology exists that is capable of obtaining globally optimum MEMS.

This paper presents a novel methodology that realizes the cooptimization of the MEMS sensor and the read-out circuitry for optimal mixed-domain system performance. The feasibility of the approach is demonstrated by single-objective and multi-objective optimizations of a capacitive MEMS accelerometer, which includes both mechanical and electronic domains. The paper aims to show that MEMS sensor/circuit co-optimization is possible. Obviously, the details of the methodology depend on the MEMS 
element used, although the general approach is the same. Thus, a model has to be derived for every MEMS component considered. Also, the parameters of interest are very diverse and vary from MEMS device to MEMS device. Hence, we will concentrate on accelerometer example here.

The paper is organized as follows. Section II reviews the heterogeneous MEMS device to optimize, including the details of the sensor model, capacitive sensor interface, the circuit topology, and system-level design variables. Section III introduces the proposed co-optimization technique. Experimental results and a comparison to conventional top-down design approaches are shown in Section IV. The experimental results of bi-objective optimization of the MEMS accelerometer are given in Section V. Limitations of the composition of independently optimized parts are also illustrated. Finally, Section VI concludes the paper.

\section{MEMS ACCELEROMETER SYSTEM}

The accelerometer system topology used for demonstration of the design methodology is a combination of a capacitive sensor [4] and a transimpedance amplifier based read-out stage [9].

\section{A. MEMS Sensor}

The MEMS sensor topology has two fixed and one moving electrodes. Two different gaps at the top and bottom sides create a differential capacitance pair with respect to the acceleration direction; hence, a voltage signal can be generated from the related capacitance changes. The MEMS device topology given in Figure 1 involves several fabrication steps like wafer-to-wafer bonding, vacuum packaging, and deep material etching. Figure 2 shows the moving mass, which is the middle electrode of the capacitive MEMS accelerometer sensor.

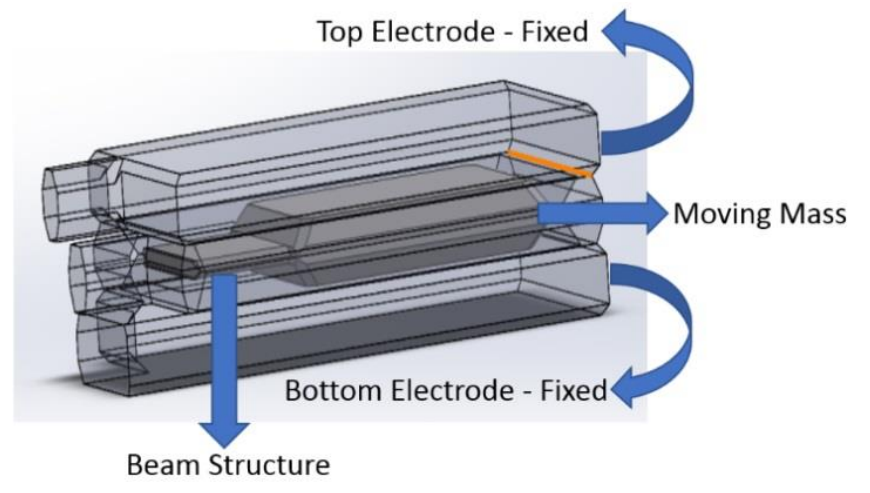

Figure 1. The MEMS capacitive accelerometer

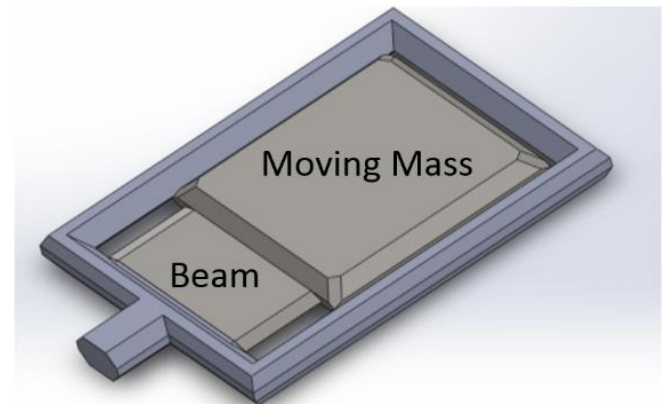

Figure 2. The moving mass of the MEMS device

It is not feasible to run MEMS device simulations within an iterative optimization loop since a single device simulation using e.g., the MEMS module of the multiphysics simulation package Comsol [10] takes around ten minutes. Considering that the number of simulations in a typical evolutionary optimization algorithm will be $N \cdot G$, where $N$ is the population size and $G$ is the number of generations, the overall number of MEMS simulations is quite high, resulting in an impractical computation time. To solve this problem, highly accurate, well-known analytical models (under vacuum conditions) can be used $[9,11$ $13]$.

In order to calculate the spring constant, the displacement on the spring side of the mass (per $a$ accelaration) has to be calculated first. As it can bee seen in Figure 1, the mass is carried by a single cantilever beam and the entire load is at the free end of the beam. The deflection at the free end of the beam in such topologies can be calculated with the analytical model [11-13]:

$$
\operatorname{disp} 1=\frac{4 \cdot m \cdot a \cdot L_{\text {beam }}{ }^{3}}{W_{\text {beam }} \cdot t_{\text {beam }}{ }^{3} \cdot E}
$$

disp 1 is the displacement at the free end of the beam, $m$ is the overall mass carried by the beam, $a$ is the acceleration applied, $E$ is the Young's modulus of the material used, $W_{\text {beam }}, L_{\text {beam }}$ and $t_{\text {beam }}$ are the width, length and thickness of the sensor beam.

After the calculation of the displacement, the spring constant of the beam structure can be calculated as:

$$
k=\frac{m \cdot a}{\operatorname{disp} 1}
$$

By using the spring constant and the mass, the resonant frequency of the system is [9]:

$$
\omega_{r}=\sqrt{\frac{k}{m}}
$$

The analytical model of the Brownian sensor noise [9], caused by the random collision of air molecules with the sensor, is: 


$$
\sqrt{\frac{a^{2}}{\Delta f}}=\frac{\sqrt{4 \cdot k_{b} \cdot T \cdot \omega_{r}}}{m \cdot Q}
$$

It can be seen that the noise is a function of the temperature $T$, the resonant frequency of the device $\omega_{r}$, the Boltzmann constant $k_{b}$, the overall system mass $m$ and the quality factor $Q$, which is determined by the damping level of the system, hence, the vacuum packaging technology used. Selecting $Q=500$ is a practical assumption since most of the packaging tools can achieve such good vacuum levels.

The last equation used here evaluates the bending angle of the spring mass system, $\theta$, to calculate the displacement at the other side of the mass as well, which allows the calculation of both top and bottom capacitances for the moving mass structure. Calculation of the bending angle is based on the small angle approximation [11] since the displacement itself is very small comparing to the length of the beam structure:

$$
\theta=\frac{\operatorname{disp} 1}{L_{\text {beam }}}
$$

The accuracy of the noise model has been verified by using two different commercial sensors with the same topology and material properties that have been used for all the experiments in this paper. Both devices have been simulated using the Comsol Multiphysics MEMS Module, with stationary type of study, and the simulation results for the displacement of the moving mass have been used to calculate first the spring constant in (2), and the resonant frequency in (3), and, then, the noise using the analytical model in (4).

Comparisons between the analytical model of the noise and the noise values obtained from the device datasheets, which refer to actual analog noise measurements, are shown in Table 1. The analytical model errors for the two devices are $1.3 \%$ and $1.8 \%$. The corresponding spring constant errors for these two simulations are $1.7 \%$ and $3.3 \%$, respectively, and the actual values are given in Table 2.

Table 1. Calculated and datasheet noise for the commercial devices

\begin{tabular}{|c|c|c|}
\hline Device & Analytical Model & Datasheet \\
\hline Commercial \#1 & $18.2(\mu \mathrm{g} / \sqrt{\mathrm{Hz}})$ & $18.0(\mu \mathrm{g} / \sqrt{\mathrm{Hz}})$ \\
\hline Commercial \#2 & $12.6(\mu \mathrm{g} / \sqrt{\mathrm{Hz}})$ & $12.4(\mu \mathrm{g} / \sqrt{\mathrm{Hz}})$ \\
\hline
\end{tabular}

Table 2. Calculated and simulated spring constants using displacement results

\begin{tabular}{|c|c|c|}
\hline Device & Simulated & Analytical Model \\
\hline Commercial \#1 & $7.3(\mathrm{kN} / \mathrm{m})$ & $7.4(\mathrm{kN} / \mathrm{m})$ \\
\hline Commercial \#2 & $15.4(\mathrm{kN} / \mathrm{m})$ & $14.9(\mathrm{kN} / \mathrm{m})$ \\
\hline
\end{tabular}

For additional verification of the accuracy of the spring constant model, 32 different Comsol simulations with a uniformly distributed set of design variables have been carried out. The spring constant model, as given in (2) above, is a function of all three dimensions of both the mass and the beam structure. Twelve simulations, including the minimum and maximum values of these 6 design variables as well as the exact dimensions of the two commercial sensors were performed. Another 18 simulations with randomly selected dimensions were executed in order to cover the entire range of the design parameters considered in the optimization loop. The reason for these extra verifications is that the genetic operators might come up with some solutions whose dimensions are quite different from those of the commercial devices.

Displacements with respect to the acceleration have been obtained via Comsol simulations and the same displacements have been calculated using the analytical model. Table 3 shows the minimum, maximum and mean errors of the 32 MEMS device evaluations while Figure 3 shows all 32 spring constant values obtained by Comsol simulations and the analytical model.

Table 3. Analytical model error for the spring constant

\begin{tabular}{|c|c|c|}
\hline Mean Error & Maximum Error & Minimum Error \\
\hline $1.3 \%$ & $6.5 \%$ & $0.1 \%$ \\
\hline
\end{tabular}

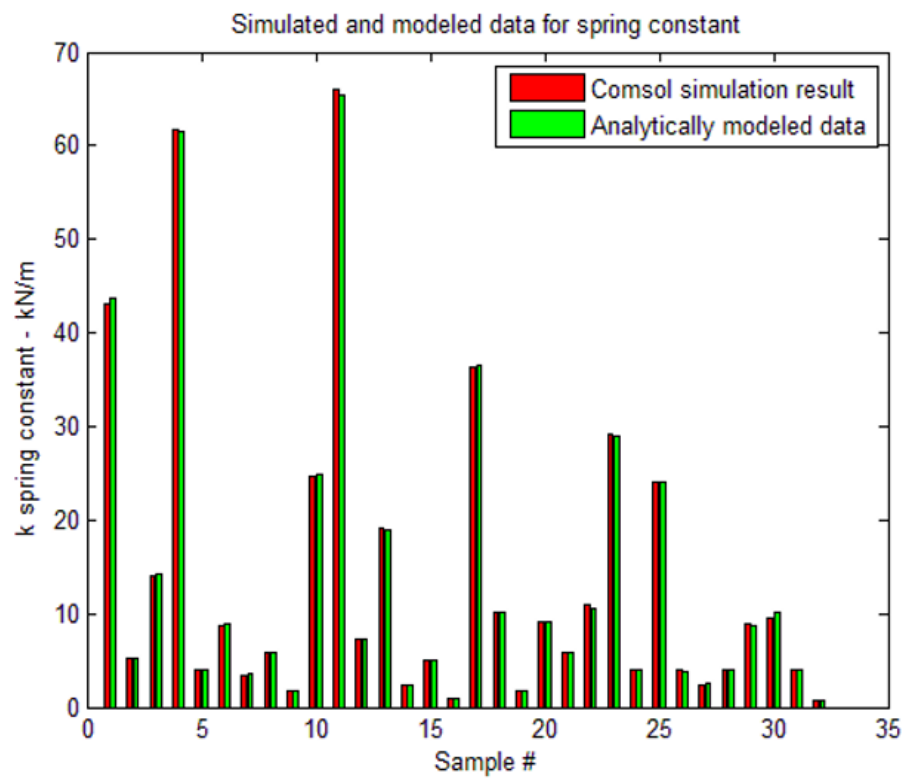

Figure 3. Simulated and modeled data for the spring constant

Besides the implementation of the analytical model, the sensor interface for the circuit-level simulations has also been developed [9]. Figure 4 shows the equivalent electrical circuit of the sensor. The sensor equivalent circuit consists of two capacitances that are calculated via the sensor analytical model, as well as the sensor noise at the circuit's input node. 


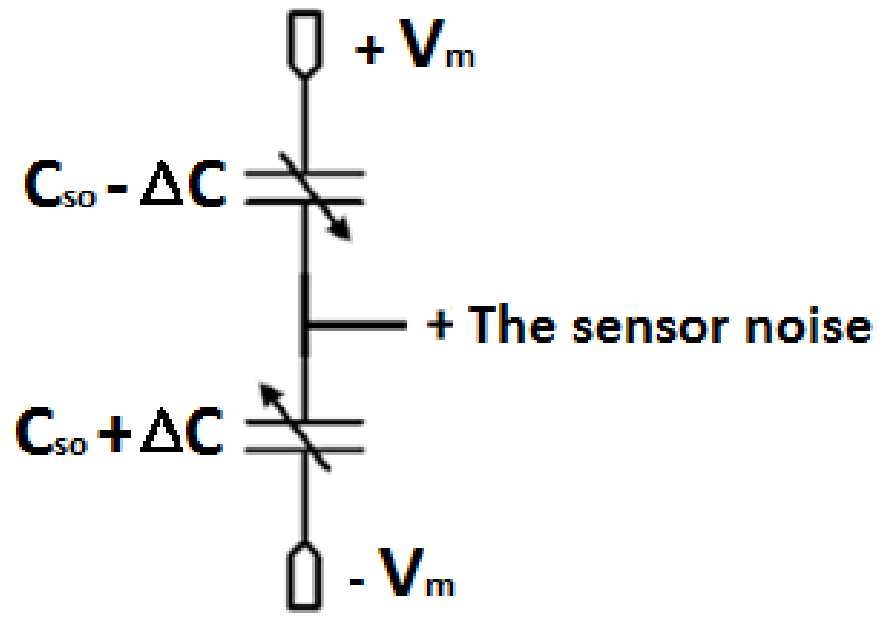

Figure 4. The equivalent circuit used for the MEMS accelerometer sensor

\section{B. C/V Converter Topology}

The topology selected for the capacitance-to-voltage (C/V) converter to measure the capacitive changes on the MEMS capacitive electrodes is a conventional transimpedance amplifier (TIA)-based converter with a resistance and capacitance feedback. The system design, including the MEMS element and the amplifier, is given in Figure 5. The capacitive change at the MEMS device creates a sensing current that is converted into a voltage value at the output of the amplifier.

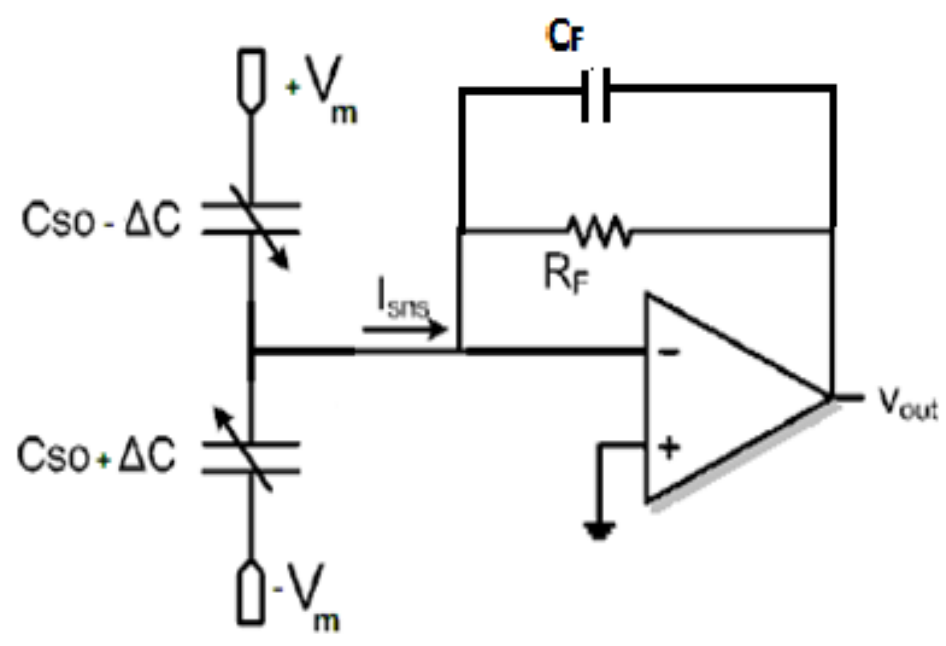

Figure 5. MEMS accelerometer with the TIA-based C/V converter

\section{System Level Design Variables}

In order to synthesize the MEMS accelerometer, several design variables from the different building blocks have been used. These design variables are: the three dimensions of the mass and the beam structures of the sensor, the capacitive gap between the moving mass and the fixed electrodes, the bias current and the transistor widths and lengths of the amplifier also the feedback resistor and the capacitor of the $\mathrm{C} / \mathrm{V}$ converter. The allowable ranges for the design variables are given in Table 4.

Table 4. Allowable ranges of the design variables

\begin{tabular}{|c|c|c|}
\hline Design Variable & Minimum Value & Maximum Value \\
\hline$L_{\text {mass }}, W_{\text {mass }}, W_{\text {beam }}$ & $200 \mu \mathrm{m}$ & $3 \mathrm{~mm}$ \\
\hline$t_{\text {mass }}$ & $250 \mu \mathrm{m}$ & $500 \mu \mathrm{m}$ \\
\hline$L_{\text {beam }}$ & $200 \mu \mathrm{m}$ & $2 \mathrm{~mm}$ \\
\hline$t_{\text {beam }}$ & $50 \mu \mathrm{m}$ & $150 \mu \mathrm{m}$ \\
\hline$C_{\text {gap }}$ & $0.5 \mu \mathrm{m}$ & $1.5 \mu \mathrm{m}$ \\
\hline$W_{\text {transistors }}$ & $0.24 \mu \mathrm{m}$ & $100 \mu \mathrm{m}$ \\
\hline$L_{\text {transistors }}$ & $0.18 \mu \mathrm{m}$ & $10 \mu \mathrm{m}$ \\
\hline$R_{F}$ & $10 \mathrm{k} \Omega$ & $1 \mathrm{M} \Omega$ \\
\hline$C_{F}$ & $0.1 \mathrm{pF}$ & $10 \mathrm{pF}$ \\
\hline$I_{\text {bias }}$ & $0.5 \mu \mathrm{A}$ & $2.5 \mathrm{~mA}$ \\
\hline
\end{tabular}

Figure 6 shows the diagram amplifier to be used in the system given in Figure 5. The amplifier is in folded cascode configuration.

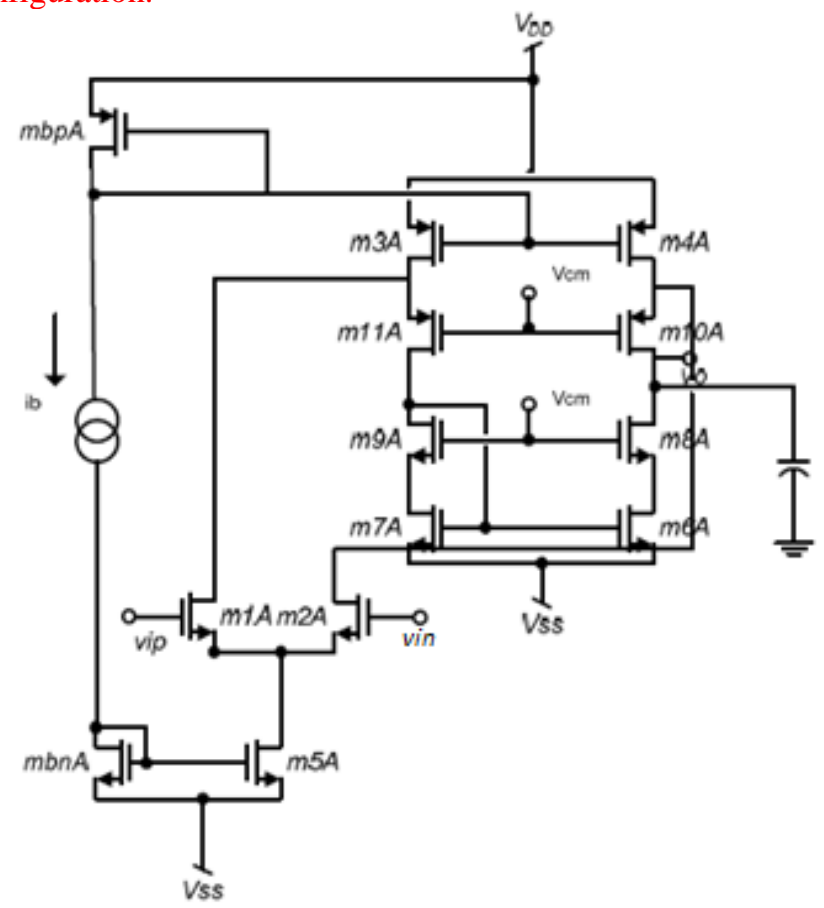

Figure 6. Folded-cascode amplifier 


\section{PROPOSED CO-OPTIMIZATION TECHNIQUE FOR THE ACCELEROMETER SYSTEM}

In order to implement an automated design methodology for the mixed-domain accelerometer, a conventional approach would be a hierarchical top-down optimization of the MEMS, where the system level synthesis is decomposed into smaller optimization subproblems like the sensor itself and the input stage electronics. The main advantage of this technique is the smaller size of the search space of each subproblem, hence, easing the convergence of optimization methods. On the other hand, the need for the partitioning of some specifications between both subproblems makes it more challenging, especially for mixed-domain systems like MEMS.

The alternative approach proposed here consists of a cooptimization process of the whole MEMS, where the sensor performance is evaluated first at each iteration of the optimization loop, and, then, these results are used for circuit level simulations, both performed within the same optimization loop. The optimal performances are obtained by using a powerful evolutionary algorithm, namely MOEA/D [14-15], that has been implemented in Matlab. The proposed technique is expected to bring solutions to the specification partitioning problem since such partitioning disappears from the design process. It should also be noticed that thanks to the co-optimization, problems related to composition of separately optimized sensor and circuitry are avoided.

The proposed co-optimization methodology requires evaluations at both, the circuit and the sensor level. At the circuit level, simulations are performed using Hspice, while the MEMS performance evaluations are realized using the accurate analytical models of the MEMS sensor described in Section II in order to decrease the optimization time required. The evaluation flow of the proposed methodology is illustrated in Figure 7.

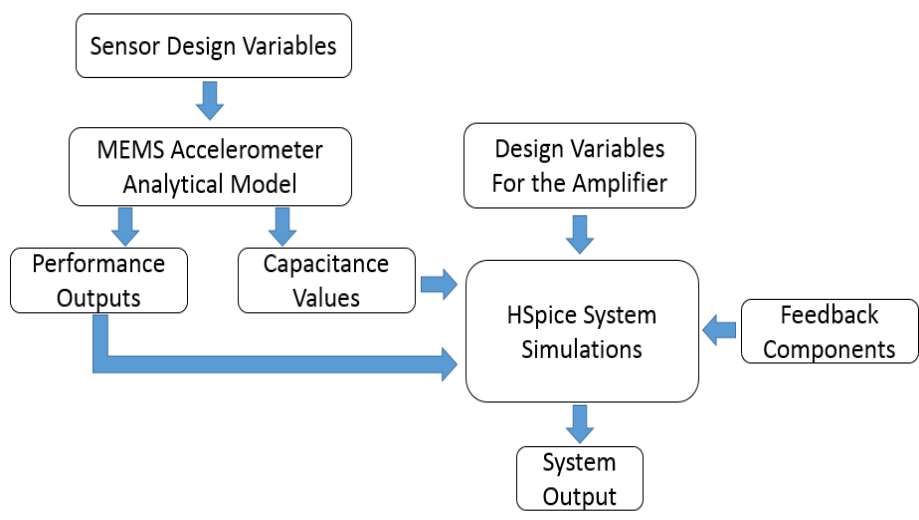

Figure 7. Evaluation flow used for MEMS accelerometer system synthesis

In a single iteration of the optimization loop, first, the MEMS analytical model is evaluated using the values of the design variables dedicated to the MEMS sensor. The performance outputs such as area and noise are extracted from the MEMS model evaluations. The model is also used to obtain the capacitive interface of the MEMS sensor to be adapted to the Hspice netlist of the read-out circuitry, in order to simulate the system level performance outputs. The design variables dedicated to the electrical simulations are introduced at this level as well. Once the system level performance values are obtained, the evolutionary algorithm uses these outputs, as well as the design variables providing these performances, in order to improve the quality of the system design in the following iteration.

In order to demonstrate the proposed technique, the MEMS accelerometer system given in Section 2 has been synthesized. Figure 8 shows the flow diagram of a single iteration of the proposed optimization methodology for this mixed-domain MEMS accelerometer. For the implementation of the optimization loop, first, the system design variables are determined by the optimization algorithm. Then, the design parameters of the MEMS sensor are used for the evaluations of the MEMS analytical models. After the evaluation of the MEMS analytical model, the capacitive interface for the circuit simulations (bottom and top capacitances per $g$ acceleration) as well as the sensor noise are obtained and integrated into the evaluations of the system level that are performed with Hspice. The area of the MEMS sensor is another output of the MEMS model for the calculation of the system level manufacturing cost. The measurement range of the sensor is also obtained.

After the evaluation of the MEMS sensor using analytical models, system level simulations using Hspice take place. The design parameters of this second phase are the design parameters of the amplifier, the feedback components and the integrated sensor outputs as mentioned above.

Generation of the system level design variables with MOEA/D

(Sensor design variables, circuit design variables and feedback components)

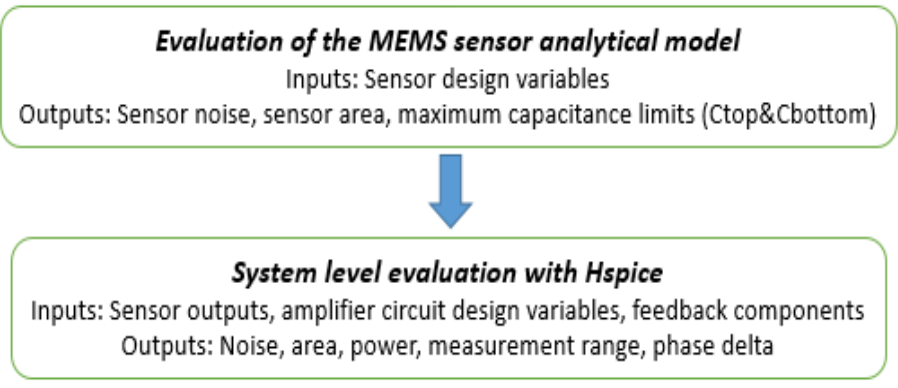

Figure 8. Evaluation of a single iteration in MEMS accelerometer synthesis using the proposed methodology 
From the circuit level simulations, the system level outputs, i.e, the measurement range, phase delta, system noise, total system cost and the power consumption, are obtained. Depending on the user selection, these system performances can either be selected as objectives or constraints.

\section{COMPARISON TO ALTERNATIVE TOP-DOWN DESIGN TECHNIQUES}

The co-optimization of the MEMS accelerometer proposed in this paper is expected to avoid some significant drawbacks of alternative design methodologies. In particular, the cooptimization of the sensor and the circuit is expected to result in optimal indirect partitioning of the MEMS specifications between both, the sensor and the circuit. To demonstrate this, the proposed methodology will be compared with a top-down design methodology in which the sensor is optimized first, and, then, the circuitry is optimized for this optimal sensor. This philosophy emulates some reported approaches e.g., that used in [8]. In this methodology, the user-defined partitioning of specifications between sensor and surrounding circuitry may result in suboptimal system design, even though the sensor and the circuit can be individually optimal. Let us consider the single-objective optimization, using the differential evolution algorithm, of the MEMS accelerometer system. The optimization was run with a population of 100 vectors (solutions) along 100 iterations.

Table 5 shows the system level specifications. The total manufacturing cost of the MEMS system is the minimization objective while the other performance outputs are handled as constraints.

The total system cost in USD is given by:

$$
\text { total }_{\text {cost }}=0.58 \cdot\left(\text { Area }_{t r}+\text { Area }_{f b}\right)+0.16 \cdot \text { Area }_{M E M S}+2
$$

This equation assumes a hybrid manufacturing approach in which the MEMS sensor and the circuit including the feedback components are manufactured separately and, then, bonded. Hence, different coefficients, determined by the manufacturing costs per area for each related technology, appear at equation (6). The coefficients 58 cents $/ \mathrm{mm}^{2}$ and 16 cents $/ \mathrm{mm}^{2}$ were obtained from the Tubitak Turkey cleanroom facilities. These coefficients are the cost per area for standard CMOS (including passive components) and MEMS manufacturing, respectively. The big difference between them mostly comes from the number of masks needed, which makes the complexity of the process flow for CMOS and MEMS manufacturing quite different. The additional two dollars in (6) correspond to the fixed packaging cost, independent of the specific MEMS design.
Table 5. System level specifications for single-objective optimization

\begin{tabular}{|c|c|}
\hline Performance - System & Value \\
\hline Overall System Cost $($ USD) & Minimize \\
\hline Total System Noise $(\mu \mathrm{g} / \sqrt{ } \mathrm{Hz})$ & $<8 / 12.5 / 25 / 50 / 80$ \\
\hline Phase Delta $\left({ }^{\circ}\right)$ & $<3$ \\
\hline Measurement range $(\mathrm{g})$ & $>100$ \\
\hline Power Consumption $(\mu \mathrm{W})$ & $<60$ \\
\hline
\end{tabular}

For a significant comparison of the two methodologies, five different optimizations for five different noise specifications, all aimed at minimizing the overall system cost, have been run, as shown in Table 5. The other constraints are the acceleration measurement range of the MEMS device, overall power consumption and phase delta, which is the phase difference of the system output from 90 degrees. The phase delta at the output of the $\mathrm{C} / \mathrm{V}$ converter is an important quality parameter for the MEMS device since the output signal is likely to be demodulated. All the constraints, except noise, are the same for all optimizations. The limit value for the phase delta, or phase error has been selected as 3 degrees, which is a typical value in MEMS accelerometer designs [16]. The power consumption constraint, on the other hand, has been selected as $60 \mu \mathrm{W}$. The power consumption of the read-out circuitry may span from a few hundred $\mu \mathrm{Ws}$ [17] to few $\mathrm{mWs}$ [18]. Since the read-out circuitry used only has a single stage that works as a $\mathrm{C} / \mathrm{V}$ converter, the constraint has been set to low values to enable low power designs where the circuit noise will have an impact on the system level noise as well.

The system objectives and constraints given above can be directly handled in the proposed co-optimization approach. However, the conventional top-down approach optimizes the MEMS sensor first, and, then, sizes the circuit using the optimal MEMS sensor. It is obvious that cost minimization should also be an objective of both the individual sensor and the circuit optimizations. However, noise is a challenging constraint since it is introduced into the system by both, the sensor and the readout circuitry. Therefore, the system noise specification in Table 5 must be first partitioned between the sensor and the circuit. For that purpose, experiments with five different partitionings, shown in Table 6, have been executed for each of the five noise specifications in Table 5, resulting in the formulation of 25 optimization problems in total. Practically, the contribution of the sensor noise to the overall system noise is expected to be higher than the contribution of the read-out circuitry. The sensor signal itself is noisy and the interface should not add an appreciable amount of noise. Hence, the circuit noise should be kept small. The choice of the partitioning values are based on this assumption.

Another constraint is the minimum measurement range that the system can achieve. Both, the sensor and the circuit have direct impact on the measurement range through different mechanisms. For the MEMS sensor, the acceleration range is limited by the movement capabilities of the mass, hence, determining the capacitive gaps of the device. The movement of the mass towards 
the fixed electrodes might cause sticking issues. The measurement range limitation on the circuit side is a different mechanism that is determined by the supply voltage and the amplification level of the input signal. With a high level of amplification, the input signal, that was generated by the changing sensor capacitances, is likely to saturate at the system output. That may result in the impossibility to measure the acceleration values that are lower than the measurement range constraint.

The other constraints: phase delta and power consumption, are only determined by the circuit. Hence, these constraints are only applied during the optimization of the circuit that interfaces the optimal MEMS sensor. Tables 7 and 8 show the objective and the design constraints used during the isolated optimization of the MEMS sensor and circuitry, respectively, in the top-down design approach.

Table 6. Partitioning of noise constraint among the MEMS sensor and the circuit

\begin{tabular}{|c|c|c|}
\hline Partitioning & Sensor noise & Circuit noise \\
\hline Partitioning \#1 & $50 \%$ & $50 \%$ \\
\hline Partitioning \#2 & $60 \%$ & $40 \%$ \\
\hline Partitioning \#3 & $70 \%$ & $30 \%$ \\
\hline Partitioning \#4 & $75 \%$ & $25 \%$ \\
\hline Partitioning \#5 & $80 \%$ & $20 \%$ \\
\hline
\end{tabular}

Table 7. Minimization objective and design constraints of the MEMS sensor

\begin{tabular}{|c|c|}
\hline $\begin{array}{c}\text { Performance }- \text { MEMS } \\
\text { Sensor }\end{array}$ & Value \\
\hline Overall System Cost $(\mathrm{USD})$ & Minimize \\
\hline Sensor Noise $(\mu \mathrm{g} / \sqrt{\mathrm{Hz}})$ & Depends on the partitioning \\
\hline Measurement range $(\mathrm{g})$ & $>100$ \\
\hline
\end{tabular}

Table 8. Minimization objective and design constraints of the C/V converter

\begin{tabular}{|c|c|}
\hline Performance - Circuitry & Value \\
\hline Overall System Cost $(\mathrm{USD})$ & Minimize \\
\hline Circuit Noise $(\mu \mathrm{g} / \sqrt{\mathrm{Hz}})$ & Depends on the partitioning \\
\hline Phase Delta $\left({ }^{\circ}\right)$ & $<3$ \\
\hline Measurement range $(\mathrm{g})$ & $>100$ \\
\hline Power Consumption $(\mu \mathrm{W})$ & $<60$ \\
\hline
\end{tabular}

Table 9 shows the fabrication cost results of the proposed optimization methodology for the five different synthesis problems proposed in Table 5 as well as the 25 experimental results of the conventional top-down methodology for the same five synthesis problems (five different noise constraints in the first column in Table 9) but considering the five different partitioning solutions in Table 6 for each of them. It should be noticed that these results do not include the packaging cost given in (6).

As Table 9 demonstrates, the proposed methodology, as a cooptimization of the sensor and the circuit, yields better results than the alternative top-down design methodology. Isolated optimization of the MEMS sensor first, and then the circuit optimization using the pre-optimized sensor, on the other hand, requires partitioning among some specifications like noise, for which both the MEMS and the circuit have impact on the final system performance. It can be observed that the optimal partitioning in the conventional top-down approach is different for each noise specification. But even for the best partitioning among five different possibilities, the lowest system cost obtained is higher than the proposed optimization technique for all five different system noise specifications.

Table 9. Single-objective optimization results of the fabrication cost (in USD) for both the proposed optimization methodology and the conventional top-down design approach using the noise partitionings in Table 6

\begin{tabular}{|c|c|c|c|c|c|c|}
\hline $\begin{array}{c}\text { Noise } \\
\text { Constraint } \\
\boldsymbol{\mu g} / \sqrt{\mathbf{H z}}\end{array}$ & $\begin{array}{c}\text { Cost } \\
\text { Proposed } \\
\text { Approach }\end{array}$ & $\begin{array}{c}\text { Cost } \\
\text { Part.1 }\end{array}$ & $\begin{array}{c}\text { Cost } \\
\text { Part.2 }\end{array}$ & $\begin{array}{c}\text { Cost } \\
\text { Part.3 }\end{array}$ & $\begin{array}{c}\text { Cost } \\
\text { Part.4 }\end{array}$ & $\begin{array}{c}\text { Cost } \\
\text { Part.5 }\end{array}$ \\
\hline 8 & 1.51 & 1.67 & 1.52 & 1.71 & 1.79 & 1.89 \\
\hline 12.5 & 0.99 & 1.24 & 1.09 & 1.06 & 1.14 & 1.21 \\
\hline 25 & 0.55 & 0.79 & 0.71 & 0.64 & 0.56 & 0.63 \\
\hline 50 & 0.32 & 0.50 & 0.44 & 0.39 & 0.36 & 0.33 \\
\hline 80 & 0.24 & 0.41 & 0.37 & 0.32 & 0.30 & 0.28 \\
\hline
\end{tabular}

\section{MULTIOBJECTIVE MEMS SYNTHESIS}

Two-dimensional optimizations of the capacitive MEMS accelerometer have been run using the proposed synthesis approach. The overall system noise and the manufacturing cost have been selected as the design objectives. Design constraints and objectives are listed in Table 10. The population size and the number of generations of the optimization algorithm are 200 and 300 , respectively. The only difference of the constraints compared to the single-objective optimization is the power consumption, that was set to $300 \mu \mathrm{W}$, as a practical design value [17].

Table 10. System specifications and optimization parameters for two-dimensional synthesis of the MEMS system

\begin{tabular}{|c|c|}
\hline Performance & Value \\
\hline Overall System Cost $(\mathrm{USD})$ & minimize \\
\hline System Noise $(\mu \mathrm{g} / \sqrt{\mathrm{Hz}})$ & minimize \\
\hline Phase Delta $\left(^{\circ}\right)$ & $<3$ \\
\hline Measurement range $(\mathrm{g})$ & $>100$ \\
\hline Power Consumption $(\mu \mathrm{W})$ & $<300$ \\
\hline
\end{tabular}

Figure 9 shows an approximation to the two-dimensional Pareto Front (PF), which includes the solutions trading-off system noise and manufacturing cost. Three snapshots for different number of generations in Figure 9 allow to monitor the evolution of the Pareto front. 


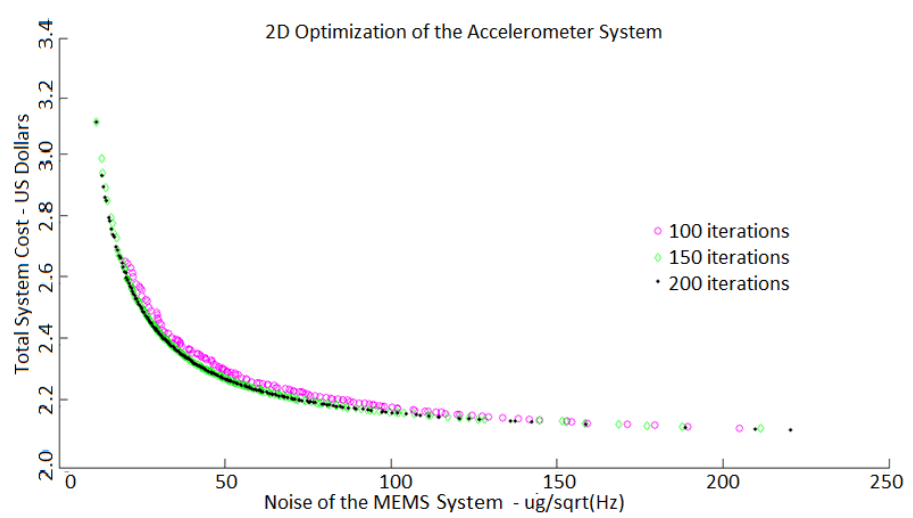

Figure 9. The Pareto front of two-dimensional optimization

The evolution of the Pareto front suggests that the optimization convergence is practically stalled by the $150^{\text {th }}$ iteration since there is no significant improvement afterwards. In order to quantitatively check the level of convergence along different iterations, the Lebesgue measure [19], also known as hypervolume, has been plotted vs. the number of iterations in Figure 10. The results suggest that the optimization process does not significantly improve the results after around the $110^{\text {th }}$ iteration.

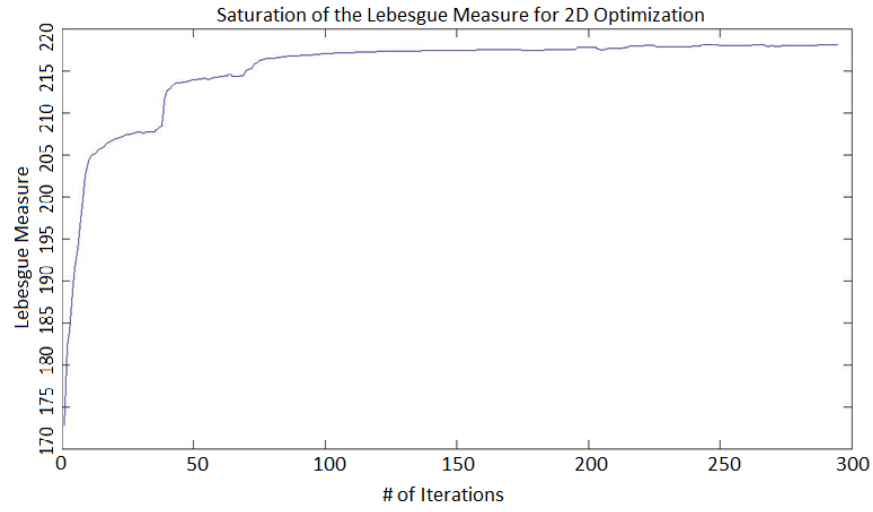

Figure 10. The Lebesgue measure of different iterations in two-dimensional optimization

In the proposed optimization of the mixed-domain MEMS accelerometer, not only the system-level results, but also the sensor and the circuit are expected to be individually optimal. In order to illustrate that, two different PFs have been obtained. The first one is the PF obtained by applying a similar optimization approach but only focused on the MEMS sensor. The second PF is the MEMS sensor front that is obtained by extracting the MEMS sensor designs from the mixed-domain optimization of the whole MEMS accelerometer in Figure 9. Figure 11 shows that the optimized MEMS sensor PF and the extracted MEMS sensor PF have very similar performances. This suggests that each optimal
MEMS accelerometer design is obtained by using a MEMS sensor with some optimal trade-off among its performances.

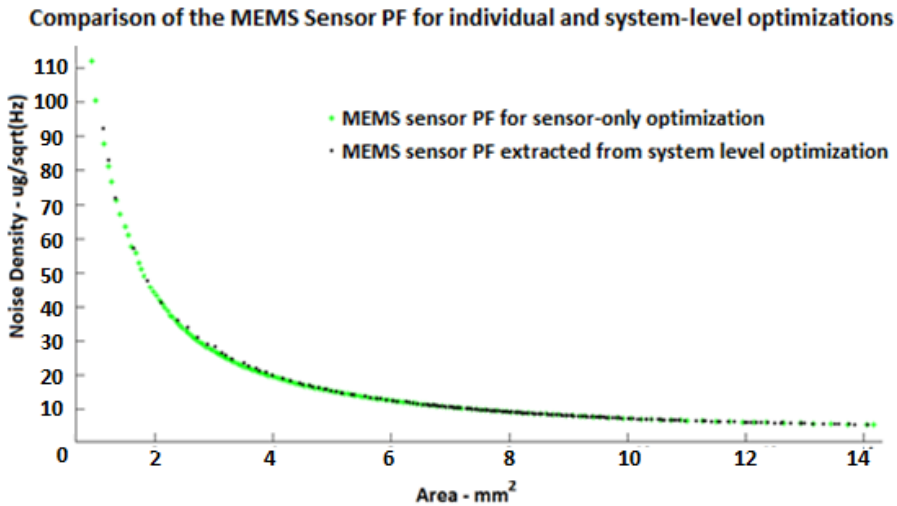

Figure 11. Comparison of the MEMS sensor PF for individual and system-level optimizations

In order to assess the accuracy of the sensor model on the final optimization results, eleven uniformly distributed design points have been extracted from the system-level Pareto front in Figure 9. The selected MEMS have been simulated using Comsol, in order to compare the simulation results with the analytically calculated spring constants. The results of the model accuracy for each MEMS design point is listed in Table 11. This table also shows the deviation on the MEMS system-level noise that is caused by the errors at the spring constant calculation. The highest noise deviation on the Pareto front is less than $2 \%$. On the other hand, the calculation of the second objective, the total system cost, is fully accurate since it only relies on the calculation of area.

Table 11. Errors of spring constant and total noise for 11 different designs of the MEMS POF.

\begin{tabular}{|c|c|c|}
\hline $\begin{array}{c}\text { Design Point on PF } \\
\text { (Solution \#) }\end{array}$ & $\begin{array}{c}\text { Error at spring } \\
\text { constant k }(\%)\end{array}$ & $\begin{array}{c}\text { Error at the MEMS } \\
\text { Noise (\%) }\end{array}$ \\
\hline 1 & 1.65 & 0.82 \\
\hline 20 & 3.82 & 1.89 \\
\hline 40 & 0.88 & 0.44 \\
\hline 60 & 1.12 & 0.56 \\
\hline 80 & 2.80 & 1.39 \\
\hline 100 & 2.65 & 1.32 \\
\hline 120 & 0.45 & 0.22 \\
\hline 140 & 1.82 & 0.91 \\
\hline 160 & 3.04 & 1.51 \\
\hline 180 & 2.12 & 1.05 \\
\hline 200 & 3.66 & 1.81 \\
\hline
\end{tabular}

It might seem a priori that the Pareto front in Figure 9 could also be obtained by composing the MEMS sensor Pareto front with the Pareto front of the readout circuit. Let us consider that for the capacitive MEMS accelerometer system defined in Section II, Pareto fronts for the MEMS sensor and the circuitry, involving minimization of cost and noise, have been independently generated. Composing the design points from each Pareto front in 
order to achieve optimal system level cost and noise is, a priori, feasible. However, that might result in a constraint violation at the system level due to dependence of the TIA feedback network from the selection of the capacitive MEMS interface. Moreover, changing the values of the capacitive interface might result in a change of the noise calculations at the circuit level, inducing a performance shift. These problems are overcome by mixeddomain co-optimization as the proposed technique performs. In order to demonstrate the risk of constraint violation and performance shift, several experiments have been performed.

In order to optimize the MEMS accelerometer with the specifications given in Table 10 by composing individually optimized sensor and circuit, Pareto fronts have been separately generated using the sensor specifications in Table 12 and the circuit specifications for a standard capacitive interface in Table 13. For both optimizations, manufacturing cost and noise have been used as objectives, while the constraint selection depends on the impact on the system level performance. For example, the measurement range is affected by both of the sensor and the readout circuitry devices while the power consumption is a contribution of the electronic circuit only. The population size for sensor and circuit optimizations is 200. For both optimizations, the number of generations is 300 .

Table 12. Objectives and constraints for two-dimensional optimization of the MEMS sensor

\begin{tabular}{|c|c|}
\hline Parameter / Performance & Value \\
\hline MEMS Sensor Cost $(\mathrm{USD})$ & Minimize \\
\hline MEMS Noise $(\mu \mathrm{g} / \sqrt{\mathrm{Hz}})$ & Minimize \\
\hline Measurement range $(\mathrm{g})$ & $>100$ \\
\hline
\end{tabular}

Table 13. Objectives and constraints for two-dimensional optimization of the MEMS read-out circuitry

\begin{tabular}{|c|c|}
\hline Parameter $/$ Performance & Value \\
\hline Circuit Cost $(\mathrm{USD})$ & Minimize \\
\hline Circuit Noise $(\mu \mathrm{g} / \sqrt{\mathrm{Hz}})$ & Minimize \\
\hline Phase Delta $\left({ }^{\circ}\right)$ & $<3$ \\
\hline Measurement range $(\mathrm{g})$ & $>100$ \\
\hline Power Consumption $(\mu \mathrm{W})$ & $<300$ \\
\hline
\end{tabular}

In order to demonstrate the risks of composing separately designed mixed-domain devices, 50 different MEMS sensor designs have been composed with the same optimal circuit design. The MEMS sensor Pareto front used is the front given in Figure 11 , where 50 uniformly distributed sensor designs have been selected for composing with a single circuit. The selected circuit design, on the other hand, has a noise of $5 \mu \mathrm{g} / \mathrm{JHz}$ and a total cost of 0.16 USD without packaging cost. One of these 50 compositions resulted in a system-level constraint violation, while five of them caused performance shift. The other 44 , on the other hand have no such drawbacks but result in non-optimal design points as expected.
As the results suggest, the composition of separately designed MEMS mechanical sensor and the read-out circuitry in the electronic domain has some drawbacks that the nature of the proposed mixed-domain optimization has not. Hence, besides the optimal partitioning advantage, the proposed optimization methodology can also overcome the problems introduced by the composition of the mixed-domain devices.

\section{CONCLUSION}

The paper proposes a novel methodology that realizes the cooptimization of a mixed-domain MEMS accelerometer. During the paper, alternative methodologies that can be used for the MEMS system synthesis have been developed and comparisons that demonstrate the superiority of the proposed methodology have been realized. The implemented methodology has been used for single-objective and multi-objective optimizations of a capacitive MEMS accelerometer system.

\section{REFERENCES}

[1] T. Tan, S. Roy, N. Thuy and H. T. Huynh, "Streamlining the Design of MEMS Devices: An Acceleration Sensor" , IEEE Circuits and System Magazine, pp. 18-27, 2008.

[2] Y. Zhang, R. Kamalian, A. M. Agogino, C. H. Séquin, "Hierarchical MEMS Synthesis and Optimization", Proceedings of SPIE, International society for optical engineering, vol. 5763, May 2005.

[3] R. Neul et al. "A Modeling Approach to Include Mechanical Microsystem Components into the System Simulation", Proc. Design, Automation and Test in Europe Conf., DATE, 1998

[4] M. Pak, F.V. Fernandez,G. Dundar,"Optimization of a MEMS Accelerometer Using MOEA/D Evolutionary Algorithm ”, Proc. Int. Conf. On Synthesis, modeling, analysis and simulation methods and applications to circuit design (SMACD), 2017

[5] Z. Fan et al, "Hierarchical Evolutionary Synthesis of MEMS", IEEE Congress on Evolutionary Computation, pp. 2320-2327, 2004.

[6] T. Mukherjee,G. Fedder, "Hierarchical Mixed-Domain Circuit Simulation, Synthesis and Extraction Methodology for MEMS", Journal of VLSI signal processing systems for signal, image and video technology, vol. 21, issue 3, pp. 233-249, July 1999

[7] C. Zhao, T. J. Kazmierski, "Automated Performance Optimization and Layout Synthesis of MEMS Accelerometer with Sigma-Delta Force Feedback Control Loop", Proc. IEEE Behavioral Modeling and Simulation Workshop, 2008

[8] J.Klaus, R. Paris, R. Sommer,"Systematic MEMS ASIC Design Flow using the Example of an Acceleration Sensor", Proc. Int. Conf. On synthesis, modeling, analysis and simulation methods and applications to circuit design (SMACD), 2016

[9] G. Zhang, "Design and Simulation of A CMOS-MEMS Accelerometer", PhD. Thesis, Carnegie Mellon University, 1998

[10] COMSOL Software, MEMS Module by Comsol Multiphysics GmbH, 
https://www.comsol.com/mems-module. [Accessed July 19, 2017]

[11] T. Belendez, C. Neipp, A. Belendez, "Large and small deflections of a cantilever beam", European Journal of Physics, vol. 23, no. 3, pp.371-379, May 2002

[12] J. Strong et al., "Carbon MEMS Accelerometer", Proceedings of the Comsol Conference, Boston, 2011

[13] B.E. Boser, R. T. Howe, "Surface Micromachined Accelerometers", IEEE Custom Integrated Circuits Conference, pp. 337-344, 1995

[14] B. Liu, F. V. Fernandez, Q. Zhang, M. Pak, S. Sipahi, G. Gielen, “An Enhanced MOEA/D - DE and its Application to Multiobjective Analog Cell Sizing”, IEEE Congress on Evolutionary Computation, pp. 1-7, 2010

[15] M. Pak, F. V. Fernandez, G. Dundar, “ Comparison of QMC-based Yieldaware Pareto Front Techniques for Multi-objective Robust Analog Synthesis", Integration, the VLSI Journal, vol. 55, no. 9, pp.357-365, Sept. 2016

[16] C. Acar, A. M. Shkel, "Experimental Evaluation and Comparative Analysis of Commercial Variable-Capacitance MEMS Accelerometers", Journal of Micromechanics and Microengineering, vol. 13, pp.634-645, 2003

[17] H. Song, et al., "Fully Integrated Low - Noise Readout Circuit with Automatic Offset Cancellation Loop for Capacitive Microsensors",Sensors, vol. 15 , pp. 26009-26017, 2015

[18] D. Fang, "Low Noise and Low Power Interface Circuits Design for Integrated CMOS - MEMS Inertial Sensors", Ph.D. thesis, University of Florida, 2006

[19] M. Fleischer, "The Measure of Pareto Optima. Applications to MultiObjective Metaheuristics", Conference on Evolutionary Multi - Criterion Optimization (EMO 2003), vol. 2632 of LNCS, pp.519-533, Faro, Portugal, 2003, Springer 